\title{
Spatiotemporal Memory in a Diffusion-Reaction System
}

\author{
Michael Schulz \\ Abteilung Theoretische Physik, Universität Ulm \\ D-89069 Ulm Germany* \\ Steffen Trimper, Knud Zabrocki \\ Fachbereich Physik, Martin-Luther-Universität,D-06099 Halle Germany
}

(Dated: November 1, 2018)

\begin{abstract}
We consider a reaction-diffusion process with retardation. The particles, immersed in traps initially, remain inactive until another particle is annihilated spontaneously with a rate $\lambda$ at a certain point $\vec{x}$. In that case the traps within a sphere of radius $R(t)=v t^{\alpha}$ around $\vec{x}$ will be activated and a particle is released with a rate $\mu$. Due to the competition between both reactions the system evolves three different time regimes. While in the initial time interval the diffusive process dominates the behavior of the system, there appears a transient regime, where the system shows a driveling wave solution which tends to a non-trivial stationary solution for $v \rightarrow 0$. In that regime one observes a very slow decay of the concentration. In the final long time regime a crossover to an exponentially decaying process is observed. In case of $\lambda=\mu$ the concentration is a conserved quantity whereas for $\mu>\lambda$ the total particle number tends to zero after a finite time. The mean square displacement offers an anomalous diffusive behavior where the dynamic exponent is determined by the exponent $\alpha$. In one dimension the model can be solved exactly. In higher dimension we find approximative analytical results in very good agreement with numerical solutions. The situation could be applied for the development of a bacterial colony or a gene-pool.
\end{abstract}

PACS numbers: 05.70.Ln; 82.20.-w; 05.40.-a; 87.10.+e; 87.18.Hf

*Electronic address: michael.schulz@uni-ulm.de

${ }^{\dagger}$ Electronic address: trimper@physik.uni-halle.de 


\section{INTRODUCTION}

A broad variety of different problems in physics and biology can be formulated in terms of generalized evolution equations including delay and feedback effects 1, 2]. Such memorycontrolled effects should be a further unifying feature of complex physical [3, 4] as well as biological systems [5] far from equilibrium. Recently [6] memory effects have been qualified as the key feature in describing dynamical systems. Distributed delays are able to stabilize ecological systems [7]. Even the traffic flow can be characterized by the scaling properties of the underlying memory function [8, 9]. The whole history of systems offering self-organized criticality as earthquakes is discussed in [10]. Whereas most of the papers are addressed to a purely time dependent but homogeneous memory, the present one gives an extension to spatiotemporal processes. Based on our recent studies of several evolution models [11, 12, 13, 14], where such non-Markovian memory effects had been enclosed, and on the analysis of chemical reactions with a long range memory [15], the present study is focused to a diffusion-reaction behavior with short and long range memory couplings. As an essential new element we include the case that retardation effects are relevant. Thus cells are able to interact as well as by short-range forces such as adhesion and through either long-range forces or retarded interaction via chemical signals [16]. These effects have an essential influence on the long time behavior, but they have also an impact on the dynamics in an intermediate time regime. As the consequence of the interplay between conventional diffusive transport and annihilation and creation processes with feedback we demonstrate the system evolves slowly varying plateau in the concentration.

Our analysis can be grouped into the continuing interest in feedback couplings. A memory dominated behavior is well established in analyzing the freezing processes in under-cooled liquids [18, 19], where the underlying mathematical representation is based on a projector formalism proposed by Mori 20]. As the result of the projection procedure the irrelevant degrees of freedom, which are rapid fluctuating ones, contribute to the time evolution of the relevant degrees of freedom as well as by instantaneous and by delay-controlled terms. Since the projection formalism is not restricted to selected systems, the modification of the evolution equation due to memory effects is rather generic. The relevant degrees of freedom offering a slowly varying behavior could be the concentration of certain species or the probability density for the particles. The crucial influence of memory effects can be illustrated by 
considering a single particle moving in a disordered environment. Due to the strong disorder each member of an equally prepared ensemble makes experience by its own surrounding, which is even modified by the random walk of the single particle itself. Such a behavior can be described by a non-linear Fokker-Planck equation with an additional memory term as had been demonstrated in [21]. The analytical results, based on a one-loop renormalization group analysis, is in accordance with numerical simulations [11] and further analytical studies [12]. Whereas in that approach the memory effects are believed to be originated by the inherent non-linear interaction of the many particle system themselves, i.e. the time scale of the memory is determined by the relevant variable itself, there is a broad class of models which are subjected to external delay effects [22, 23, 24]; for a survey and applications in biology, see 25].

Recently [26] memory effects in correlated anisotropic diffusion are studied in nanoporous crystalline solids. Likewise the effects of transport memory are discussed within the Fisher ${ }^{6} \mathrm{~s}$ equation [27], ratchet devises [28] also applicable for bacterial population dynamics [29]. There appear a non-linear damping and traveling wave solutions [30]. The transport with memory, depending on the survivability of a population, is analyzed in [31].

In case the transport is realized after a spatiotemporal accumulation process the time evolution of the probability or the concentration of a different species could be also dependent on the history of the sample to which it belongs. Thus the evolution of the relevant variable $p(\vec{x}, t)$ has to be supplemented by memory terms. One possible but rather general structure of an evolution equation with memory should read [32]

$$
\partial_{t} p(\vec{x}, t)=\mathcal{M}(\vec{x}, t ; p, \nabla p)+\int_{0}^{t} d t^{\prime} \int_{-\infty}^{\infty} d^{d} x^{\prime} \mathcal{K}\left(\vec{x}-\vec{x}^{\prime}, t-t^{\prime} ; p, \nabla p\right) \mathcal{L}\left(\vec{x}^{\prime}, t^{\prime} ; p, \nabla p\right)
$$

This equation is of convolution type. Whereas the operator $\mathcal{M}$ characterizes the instantaneous and local processes, both operators $\mathcal{K}$ and $\mathcal{L}$ are responsible for the delayed processes. In general the operators $\mathcal{M}, \mathcal{K}$ and $\mathcal{L}$ may be also non-linear in $p(\vec{x}, t)$ and $\nabla p(\vec{x}, t)$. They have to be specified according to the physical situation in mind. In particular we have shown [32, 33] that the form of the operator $\mathcal{L}$ is restricted when $p(\vec{x}, t)$ is assumed to be conserved. Regarding the large variety of systems with feedback couplings it seems to be worth to study simple models, which still include the dynamical features of evolution models as conservation of the relevant quantity $p(\vec{x}, t)$ and moreover, a time-delayed coupling. 
In the present paper we extend the analysis by considering another kind of memory not included in Eq. (11). To that aim let us consider a diffusion-reaction model with retardation for a concentration $p(\vec{x}, t)$. Different to Eq. (10) there appears a spatiotemporal coupling well known from retarded potentials. Each particle makes a random walk described by a diffusion equation [27] in the continuum limit. Moreover, it can be annihilated with the rate $\lambda$ spontaneously. As a competing process a self-organized creation of particles with rate $\mu$ is introduced. However the additional process is subjected to a retardation. The situation corresponds to a diffusion-reaction process where the particles are able to emit permanently signals. After a finite running time inactive particles will be excited.

\section{DIFFERENT REALIZATIONS AND THE GENERAL SOLUTION}

To be specific let us consider a diffusion-reaction model for the concentration $p(\vec{x}, t)$ of the form

$$
\begin{aligned}
\frac{\partial p(\vec{x}, t)}{\partial t} & =D \nabla^{2} p(\vec{x}, t)-\lambda p(\vec{x}, t) \\
& +\mu \int_{-\infty}^{\infty} d^{d} x^{\prime} \Phi\left(\vec{x}^{\prime}, t-\frac{\left|\vec{x}-\vec{x}^{\prime}\right|}{v}\right) N\left(\left|\vec{x}-\vec{x}^{\prime}\right|\right) \Theta\left(t-\frac{\left|\vec{x}-\vec{x}^{\prime}\right|}{v}\right)
\end{aligned}
$$

Particles, performing a random walk with diffusion constant $D$ are annihilated with the rate $\lambda$ at a certain point $\vec{x}$ at time $t$. The subsequent term describes the creation of particles with the rate $\mu$. This creation process is triggered by a signal that will be transmitted by each

particle at the time $t^{\prime}=t-\frac{\left|\vec{x}-\vec{x}^{\prime}\right|}{v}$. With other words, particles are created whenever the signal wave, originated from any point $\vec{x}^{\prime}$ at time $t^{\prime}$ is reached the point with the coordinate $\vec{x}$ at the observation time $t \leq t^{\prime}$. The quantity $\Phi\left(\vec{x}^{\prime}, t^{\prime}\right)$ is determined by the concentration $p$, and different realizations will be discussed below. To complete the model we have to fix the normalization factor $N(r)$ by $\int r^{d-1} N(r) d \Omega_{d}=1$ which leads to

$$
N(R)=\frac{1}{\Omega_{d} R^{d-1}} \quad \text { with } \quad \Omega_{\mathrm{d}}=\frac{2 \pi^{\mathrm{d} / 2}}{\Gamma(\mathrm{d} / 2)} .
$$

After Fourier transformation Eq. (2) reads

$$
\frac{\partial p(\vec{k}, t)}{\partial t}+\left[D \vec{k}^{2}+\lambda\right] p(\vec{k}, t)=\mu \Gamma(d / 2) 2^{d / 2-1} \int_{0}^{t} d t^{\prime}\left[k v ( t - t ^ { \prime } ] ^ { 1 - d / 2 } J _ { d / 2 - 1 } \left(k v\left(t-t^{\prime}\right) \Phi\left(\vec{k}, t^{\prime}\right)\right.\right.
$$


where $J_{a}(x)$ is the Bessel function. Performing Laplace transformation with respect to time we get

$$
\left(z+D k^{2}+\lambda\right) p(\vec{k}, z)=p(\vec{k}, t=0)+\frac{\mu v}{z} F\left(\frac{1}{2}, 1, \frac{d}{2} ;-\frac{k^{2} v^{2}}{z^{2}}\right) \Phi(\vec{k}, z) .
$$

Here, F(a,b,c; y) is the hypergeometric function. For the further discussion the function $\Phi(\vec{x}, t)$ has to be specified. Here we discuss three cases

$$
\begin{aligned}
\text { (i) } \Phi(\vec{x}, t) & =p(\vec{x}, 0) \delta(t) \equiv p_{0}(\vec{x}) \delta(t) \\
\text { (ii) } \Phi(\vec{x}, t) & =p(\vec{x}, t) \\
\text { (iii) } \Phi(\vec{x}, t) & =\frac{\partial p(\vec{x}, t)}{\partial t}
\end{aligned}
$$

In case (ii) the concentration is given by

$$
p(\vec{k}, z)=\frac{p_{0}(\vec{k})}{z+D k^{2}+\lambda+\frac{\mu v}{z} F\left(\frac{1}{2}, 1, \frac{d}{2} ;-\frac{k^{2} v^{2}}{z^{2}}\right)} .
$$

In case (iii) the retardation is manifested by a coupling to the time variation of the concentration itself. It results

$$
p(\vec{k}, z)=\frac{p_{0}(\vec{k})\left[1-\frac{\mu v}{z} F\left(\frac{1}{2}, 1, \frac{d}{2} ;-\frac{k^{2} v^{2}}{z^{2}}\right)\right]}{z+D k^{2}+\lambda+\frac{\mu v}{z} F\left(\frac{1}{2}, 1, \frac{d}{2} ;-\frac{k^{2} v^{2}}{z^{2}}\right)} .
$$

Both equations do not give rise to an transient regime as it will be observed in the first case. Therefore we restrict the further discussion mainly to the first case which is discussed in detail in the forthcoming section.

\section{LONG RANGE MEMORY}

In case of

$$
\Phi(\vec{x}, t)=p_{0}(\vec{x}) \delta(t)
$$

the model offers additional specifications like conservation and anomalous behavior. The realization given by the last equation means that the creation process is triggered by a signal that had been transmitted at the initial time $t=0$, where the particles are distributed with the concentration $p_{0}(\vec{x})$. The signal is presumed to be permanent existent in the whole space like the microwave background radiation in cosmology. The activation of particles by this background signal can be further generalized in the following manner. If at an arbitrary 
point $\vec{x}$ a particle is annihilated then the activation process sets in. However, to simulate the influence of the backward signal it should be restricted to a sphere with radius

$$
R(t)=v t^{\alpha}
$$

around the mentioned point at $\vec{x}$, where the exponent will be specified below. Only in case $\alpha=1$ the quantity $v$ can be interpreted as a real velocity. While the particles perform a random walk, they will be annihilated with a certain rate $\lambda$ and created with the rate $\mu$. This process is governed by signals coming from the a sphere with radius $R(t)$ around the point with the coordinate $\vec{x}$. The situation in mind is simply described by the following evolution equation

$$
\frac{\partial p(\vec{x}, t)}{\partial t}=D \nabla^{2} p(\vec{x}, t)-\int d^{d} r[\lambda p(\vec{x}, t)-\mu N(r) p(\vec{x}-\vec{r}, 0)] \delta(r-R(t)) \quad \text { with } \quad r=|\vec{r}| \text {. }
$$

Here the first term describes conventional diffusion with the diffusion constant $D$. The second term stands for the annihilation of particles with rate $\lambda$. The last terms models the situation discussed before. Particles are created with a rate $\mu$ whenever the signal wave originated from the initial state of the system is reached the spatial point $\vec{x}$. This process corresponds to a kind of self-stabilizing of the system, especially for $\lambda=\mu$, where the total particle number is conserved. If both rates are different one obtains for the total number of particles $P(t)$ the relation

$$
P(t)=P(0)\left[\frac{\mu}{\lambda}+e^{-\lambda t}\left(1-\frac{\mu}{\lambda}\right)\right]
$$

which is also independent of the realization of the function $R(t)$. In the limiting case $\lambda=\mu$ the conservation is guaranteed. In case of a vanishing rate $\lambda=0$ there occurs a linear increase of the concentration according to $P(t)=P(0)(1+\mu t)$. Let us remark that in the opposite case where growth and death rate will be interchanged, i.e. $\lambda \rightarrow-\lambda$ and $\mu \rightarrow-\mu$ the last equation leads to a complete extinction of the species after a finite time

$$
t_{e}=\frac{1}{\lambda} \ln \left(\frac{\mu}{\mu-\lambda}\right)
$$

provided $\mu>\lambda$. As one can see from Eq. (9) there appears a driving force between the concentration of the spatial point $\vec{x}$ and a point far from that at $\vec{x}-\vec{R}(t)$, i.e. all spatial points immersed in a sphere of radius $R(t)=v t^{\alpha}$ contribute to the instantaneous development of the 
system. The model describes a situation, where a set of particles performing a random walk, carry the concentration in the past like a jet. In case of zero parameter $v=0$ the system reveals an extreme long range memory, characterized by a direct coupling of the probability $p(\vec{x}, t)$ to its initial value $p(\vec{x}, 0)$. Due to this long range coupling there exits a non-trivial stationary solution as demonstrated in [17]. This stationary solution is originated by the balance of the diffusive current and the memory induced current proportional $p(\vec{x}, t)-p(\vec{x}, 0)$. In the present approach we consider a new situation consisting of the existence of an active local environment of radius $R(t)$ around each spatial point at $\vec{x}$. The situation corresponds to the presence of traps in which particles are immersed. These traps will be installed at initial time $t=0$. However the barrier to overcome is to high, so that the particles are confined within the traps. The particle inside a trap remains inactive until another particle is annihilated spontaneously at a spatial point $\vec{x}$. Let us assume that through the annihilation process energy will be released so that the particles are able to overcome the barrier of the traps and the particle included become active with a rate $\mu$ provided they are in a sphere of radius $R(t)$ around $\vec{x}$. With other word, instead of a long range memory there is a short range one induced by a coupling of the current concentration $p(\vec{x}, t)$ to the value of the distribution $p(\vec{x}-\vec{R}(t), 0)$. In that case we demonstrate that the stationary solution disappears and instead of that the system evolves a very slow decay in a broad transient time regime. In the limiting case of vanishing $v$ the stationary solution is restored. The transient regime is likewise originated due to the balance of the diffusive current and the additional memory current proportional to $p(\vec{x}, t)-p(\vec{x}-\vec{R}(t))$. Assuming the quantity $p(\vec{x}, t)$ is proportional to the probability to find the particle at the spatial point $\vec{x}$ at time $t$ we get for the mean square displacement $s(t)=\left\langle\vec{x}^{2}\right\rangle$ the evolution equation

$$
\frac{d s(t)}{d t}=2 d D-\lambda s(t)+\mu\left[s(0)+R^{2}(t)\right]
$$

In case of $R(t)=v t^{\alpha}$ the last equation can be solved resulting in

$$
s(t)=\frac{2 d D}{\lambda}\left(1-e^{-\lambda t}\right)+\mu v^{2}(-\lambda)^{1+2 \alpha} \gamma(1+2 \alpha,-\lambda t)
$$

Here $\gamma(a, y)$ is the incomplete gamma function. For nonzero $\lambda$ the asymptotic behavior of the displacement is for $\lambda t \gg 1$ given by

$$
s(t) \simeq \frac{t^{2 \alpha}}{\lambda}
$$


while in the case of vanishing annihilation rate $\lambda=0$ one gets exactly

$$
\lim _{\lambda \rightarrow 0} s(t)=2 d D t+\frac{\mu v^{2}}{1+2 \alpha} t^{1+2 \alpha} .
$$

The growth term in Eq. (2) gives rise to anomalous diffusion which dominates for $\alpha>0$ the long time behavior of the system. In particular, even for $\alpha=1 / 2$ globally the system offers a ballistic behavior. In case of conservation of $p(\vec{x}, t)$ the last relation leads to diffusion due to $\lambda=\mu$.

\section{SOLUTION}

Since the system exhibits no general analytical solution for an arbitrary behavior of $R(t)$ we restrict the further discussion to the case $\alpha=1$. Fourier transformation of Eq. (2) yields

$$
\partial_{t} p(\vec{k}, t)=-\left(D k^{2}+\lambda\right) p(\vec{k}, t)+\lambda A p_{0}(\vec{k}) \frac{J_{\frac{d-2}{2}}(k R(t))}{[k R(t)]^{\frac{d-2}{2}}} \quad \text { with } \quad A=\Gamma(d / 2) 2^{d / 2-1} ; k=|\vec{k}|
$$

Here $J_{\nu}(y)$ is the Bessel function. Although the last equation is only valid for dimension $d>1$ [38], a separate transformation reveals that the one dimensional case with

$$
\partial_{t} p(k, t)=-\left(D k^{2}+\lambda\right) p(k, t)+\mu p_{0}(k) \cos (k R(t)),
$$

is also included. To analyze whether the model offers a nontrivial stationary solution it is appropriate to consider the Laplace transformation $p(\vec{k}, z)$. Aside of special cases the transformation can be fulfilled only for $R(t)=v t$, i.e. for $\alpha=1$. From Eq. (13) we find

$$
p(\vec{k}, z)=p_{0}(\vec{k}) \frac{1+\mu / z F\left(\frac{1}{2}, 1, \frac{d}{2} ;-\frac{k^{2} v^{2}}{z^{2}}\right)}{z+D k^{2}+\lambda}
$$

where $F(a, b, c ; y)$ is the hypergeometric function. The result is always valid for $d \geq 1$. The long time limit results for $z \rightarrow 0$. Because there is no general asymptotic behavior of the hypergeometric function $F(a, b, c, y)$ for $y \rightarrow \infty$ and arbitrary dimension $d$ we will discuss different dimensions separately. 


\section{A. Exact solution for $d=1$}

In the one- dimensional case the solution for $p(x, t)$ can be expressed by elementary functions. In $d=1$ the evolution equation reads

$\frac{\partial p(x, t)}{\partial t}=D \frac{\partial^{2} p(x, t)}{\partial x^{2}}-\lambda p(x, t)+\frac{\mu}{2}[p(x-R(t), 0)-p(x+R(t), 0)] \quad$ with $\quad p(x, 0)=p_{0}(x)$,

where $R(t)=v t$ is defined by Eq. (8) with the exponent $\alpha=1$. Performing Fourier transformation with respect to the spatial coordinate we can solve the the resulting equation for $p(k, t)$. According to Eq. (14) we get

$$
p(k, t)=p_{0}(k) e^{-\left(D k^{2}+\lambda\right) t}+\frac{p_{0}(k) \mu}{2}\left(\frac{e^{i k v t}-e^{-\left(D k^{2}+\lambda\right) t}}{D k^{2}+i k v+\lambda}+c . c .\right) .
$$

By solving this equation one observes that the three terms describe three different time

regimes. Whereas the first one, as solution of the homogeneous equation, represents the initial time interval, the second term gives rise to a traveling wave behavior which tends for $v=0$ to a space dependent stationary solution. The last term is responsible for the final time interval and leads to an exponential decay. After a lengthy but straightforward backward transformation we find $p(x, t)=p_{i}(x, t)+p_{s}(x, t)+p_{f}(x, t)$ with

$$
\begin{aligned}
p_{i}(x, t) & =\frac{e^{-\lambda t}}{\sqrt{4 \pi D t}} \int d x^{\prime} p_{0}\left(x^{\prime}\right) e^{-\frac{\left(x-x^{\prime}\right)^{2}}{4 D t}} \\
p_{s}(x, t) & =\frac{2 \mu}{\Omega} \int d x^{\prime} p_{0}\left(x^{\prime}\right)\left[\Theta\left(x-x^{\prime}+v t\right) e^{-\nu_{1}\left(x-x^{\prime}+v t\right)}+\Theta\left(x^{\prime}-x-v t\right) e^{-\nu_{2}\left(x^{\prime}-x-v t\right)}\right] \\
p_{f}(x, t) & =-\frac{\mu e^{-\lambda t}}{\Omega} \int d x^{\prime} p_{0}\left(x^{\prime}\right)\left\{e^{-\nu_{1}\left(x-x^{\prime}-D t \nu_{1}\right)} \operatorname{erfc}\left(\frac{2 \nu_{1} D t-\left(x-x^{\prime}\right)}{\sqrt{4 D t}}\right)\right. \\
& \left.+e^{\nu_{2}\left(x-x^{\prime}+D t \nu_{2}\right)} \operatorname{erfc}\left(\frac{2 \nu_{2} D t+\left(x-x^{\prime}\right)}{\sqrt{4 D t}}\right)\right\}
\end{aligned}
$$

with $\Omega=\sqrt{v^{2}+4 \lambda D}, \quad \nu_{1 / 2}=\frac{\Omega \mp v}{2 D}>0$.

In the limiting case $v=0$ the part $p_{s}(x, t)$ tends to a non-trivial stationary solution

$$
p_{s}(x)=\frac{\mu}{\sqrt{\lambda D}} \int_{-\infty}^{+\infty} d x^{\prime} p_{0}\left(x^{\prime}\right) e^{-\frac{\left|x-x^{\prime}\right|}{\xi}} \quad \text { with } \quad \xi=\sqrt{\frac{D}{\lambda}} .
$$


This result is in accordance with a previous study [17]. The behavior of $p(x, t)$ is controlled by a single dimensionless parameter

$$
\kappa=\frac{v}{\sqrt{\lambda D}}
$$

which is the ratio of the two velocities $v$ and $\sqrt{\lambda D}$.

\section{B. Solution in higher dimensions}

Guided by the solution in $d=1$ let us analyze the three dimensional case. In $d=3$ the hypergeometric function in Eq. (15) can be expressed by an elementary function resulting in

$$
p(\vec{k}, z)=p_{0}(\vec{k}) \frac{1+\mu / k v \arctan \left(\frac{k v}{z}\right)}{z+D k^{2}+\lambda} .
$$

Although we are not able to get a general solution for $p(\vec{x}, t)$, one can estimate the solution in different regimes. Denote $l$ a typical length scale and $\tau$ a characteristic time scale we can distinguish two cases: (i) $v \gg \frac{l}{\tau}$ and (ii) $v \ll \frac{l}{\tau}$. Due to $\frac{k v}{z} \gg 1$ in the first case the last equation leads to

$$
p(\vec{k}, z) \approx p_{0}(\vec{k}) \frac{1+\frac{\pi \lambda}{2 k v}}{z+D k^{2}+\lambda} .
$$

From here we get an exponential decay $p(\vec{k}, t) \propto \exp \left(-\left[D k^{2}+\lambda\right] t\right)$. In more detail we obtain

$$
\begin{aligned}
p(\vec{x}, t) & \approx p_{i}+p_{f}=\frac{e^{-\lambda t}}{(4 \pi D t)^{3 / 2}} \int d^{3} x^{\prime} p_{0}\left(\vec{x}^{\prime}\right) e^{-\frac{\left(\vec{x}-\vec{x}^{\prime}\right)^{2}}{4 D t}} \\
& +\frac{\mu e^{-\lambda t}}{8 \pi v D t} \int d^{3} x^{\prime} p_{0}\left(\vec{x}^{\prime}\right) e^{-\frac{\left(\vec{x}-\vec{x}^{\prime}\right)^{2}}{4 D t}}{ }_{1} F_{1}\left(1 / 2,3 / 2 ; \frac{\left(\vec{x}-\vec{x}^{\prime}\right)^{2}}{4 D t}\right)
\end{aligned}
$$

Here ${ }_{1} F_{1}$ is the degenerate hypergeometric function which can be expressed by elementary functions: ${ }_{1} F_{1}(1 / 2,3 / 2, y)=\sqrt{\pi / 4 v} \operatorname{erfi}(\sqrt{y})[38]$. Notice that due to the condition $\frac{k v}{z} \gg 1$ the result can be not extrapolated to $v=0$. In the opposite case $\frac{k v}{z} \ll 1 \mathrm{Eq}$. (19) is rewritten in the following form

$$
\begin{aligned}
p(\vec{k}, z) & \approx \frac{p_{s}(\vec{k})}{z}+\Psi(\vec{k}, z) \quad \text { with } \\
p_{s}(\vec{k}) & =\frac{p_{0}(\vec{k}) \mu}{D k^{2}+\lambda}, \quad \Psi(\vec{k}, z)=p_{0}(\vec{k}) \frac{D k^{2}+\lambda-\mu}{\left(D k^{2}+\lambda\right)\left(z+D k^{2}+\lambda\right)} .
\end{aligned}
$$

Due to the $1 / z$ singularity in the last equation leads to a (pseudo)stationary solution $p_{s}$. which reads after Fourier transformation

$$
p_{s}(\vec{x})=\frac{\mu}{4 \pi D} \int \frac{p_{0}\left(\vec{x}^{\prime}\right)}{\left|\vec{x}-\vec{x}^{\prime}\right|} \exp \left(-\frac{\left|\vec{x}-\vec{x}^{\prime}\right|}{\xi}\right) \quad \text { with } \quad \xi=\sqrt{\frac{D}{\lambda}} .
$$


Notice that $p_{s}$ is independent of $v$. This stationary solution is in accordance with [17]. The correction to this stationary solution reads after Fourier transformation

$$
\begin{aligned}
\Psi(\vec{x}, t) & \left.=\frac{e^{-\lambda t}}{(4 \pi D t)^{3 / 2}} \int d^{3} x^{\prime} p_{0}\left(\vec{x}^{\prime}\right) \exp \left(-\left(\vec{x}-\vec{x}^{\prime}\right)^{2} / 4 D t\right)\right) \\
& +\frac{\mu}{8 \pi D} \int d^{3} x^{\prime} \frac{p_{0}\left(\vec{x}^{\prime}\right)}{\left|\vec{x}-\vec{x}^{\prime}\right|}\left\{e^{\left|\vec{x}-\vec{x}^{\prime}\right| / \xi} \operatorname{erfc}\left(\sqrt{\lambda t}+\frac{\left|\vec{x}-\vec{x}^{\prime}\right|}{\xi}\right)\right. \\
& \left.-e^{-\left|\vec{x}-\vec{x}^{\prime}\right| / \xi} \operatorname{erfc}\left(\sqrt{\lambda t}-\frac{\left|\vec{x}-\vec{x}^{\prime}\right|}{\xi}\right)\right\}
\end{aligned}
$$

With other word we find a transient behavior in an intermediate time interval, whereas in the long time limit the concentration decays to zero. Such a behavior is similar to the $\alpha$ relaxation in undercooled liquids.

For $d=2$ the hypergeometric function can be expressed by an elementary function. From Eq. (15) one gets

$$
p(\vec{k}, z)=p_{0}(\vec{k}) \frac{1+\frac{\mu}{\sqrt{z^{2}+(k v)^{2}}}}{z+D k^{2}+\lambda}
$$

Similar to the three dimensional case one has to distinguish between the limiting cases (i) $k v / z \gg 1$ and (ii) $k v / z \ll 1$. Whereas in the first case the initial and the final state $p \approx p_{i}+p_{f}$ is reflected, in the second case the solution can be approximately written as

$$
p(\vec{k}, z)=\frac{p_{s}(\vec{k}}{z}+\Psi(\vec{k}, z)
$$

As before for $v \rightarrow 0$ the system tends to a stationary solution of the form

$$
p_{s}(\vec{x})=\frac{\mu}{2 \pi D} \int d^{2} x^{\prime} p_{0}\left(\vec{x}^{\prime}\right) K_{0}\left(\frac{\left|\vec{x}-\vec{x}^{\prime}\right|}{\xi}\right) .
$$

We omit the further details. Instead of that let us consider the $d$-dimensional case. Because of the factor $1 / z$ in front of the hypergeometric function in Eq. (15) we expect the occurrence of a pseudo-stationary for arbitrary $d$. For $t \gg\left[D k^{2}+\lambda\right]^{-1}$ Eq. (13) can be solved in leading order as

$$
p(\vec{k}, t) \simeq p_{0}(\vec{k}) \lambda A \frac{J_{\frac{d-2}{2}}(k v t)}{(k v t)^{(d-2) / 2}\left[D k^{2}+\lambda\right]} .
$$

This solution can be transformed in the coordinate representation $p(\vec{x}, t)$. The result is for $0<v t<r \equiv\left|\vec{x}-\vec{x}^{\prime}\right|$

$$
p(\vec{x}, t)=\frac{\lambda \Gamma(d / 2)}{D(v t)^{(d-2) / 2} 2 \pi^{d / 2}} \int d^{d} x^{\prime} \frac{p_{0}\left(\vec{x}^{\prime}\right)}{\left(\left|\vec{x}-\vec{x}^{\prime}\right|\right)^{(d-2) / 2}} I_{\frac{d-2}{2}}(v t / \xi) K_{\frac{d-2}{2}}(r / \xi) .
$$


In the opposite case $0<r<v t$ we find

$$
p(\vec{x}, t)=\frac{\lambda \Gamma(d / 2)}{D(v t)^{(d-2) / 2} 2 \pi^{d / 2}} \int d^{d} x^{\prime} \frac{p_{0}\left(\vec{x}^{\prime}\right)}{\left(\left|\vec{x}-\vec{x}^{\prime}\right|\right)^{(d-2) / 2}} I_{\frac{d-2}{2}}(r / \xi) K_{\frac{d-2}{2}}(v t / \xi) .
$$

Here $I_{\nu}(x)$ and $K_{\nu}(x)$ are Bessel functions. In $d=1$ one gets for $t \gg\left[D k^{2}+\lambda\right]^{-1}$ the stationary solution discussed in Eq. (18).

\section{CONCLUSIONS}

In this paper we have extended the conventional diffusion reaction system by including non-Markovian memory terms. In particular the growth rate is modified accordingly. Namely the particles are permanently subjected to signals of the environment, which acts like a background radiation. In case a particles is annihilated a recreation process sets in which is triggered by this background signals from the past. Alternatively the model can be viewed as a system of traps, distributed randomly at the initial time $t=0$. The particles immersed in the traps will be activated and released from the traps only when a particle is annihilated. Due to the competition between both processes one observes different time regimes with a different behavior. In particular the system evolves a transient regime with a very slowly varying concentration field which tends under special circumstances to a stationary pattern solution in according to previous studies. Such patterns are absent for conventional diffusion in an infinite domain without boundary conditions.

\section{Acknowledgments}

This work is supported by the DFG (SFB 418). 
[1] J. D. Murray Mathematical Biology, Part II (Springer, Berlin, 2003).

[2] T. D. Franck Nonlinear Fokker-Planck Equations (Springer, Berlin, 2005).

[3] L.S.Tsimring, A.Pikovsky, Phys.Rev.Lett. 87, 250602 (2001).

[4] C.Masoller, Phys.Rev.Lett. 88, 034102 (2002); Phys. Rev. Lett. 90, 020601 (2003).

[5] M. Freeman, Nature 408, 313 (2000).

[6] A.V. Mokshin, R.M. Yulmetyev, and P. Hänggi, Phys. Rev.Lett. 95, 200601 (2005).

[7] C.W. Eurich, A. Thiel, and L. Fahse, Phys.Rev.Lett. 94, 158104 (2005).

[8] M. Treiber, D. Helbing, Phys. Rev. E 68, 046119 (2003).

[9] B.-S.K. Skagerstam and A. Hansen, Europhys. Lett. 72, 513 (2005).

[10] E. Lippiello, L.de Arcangelis, and C. Godano, Europhys. Lett. 72, 678 (2005).

[11] B. M. Schulz, S. Trimper, Phys. Lett. A 256, 266 (1999); B. M. Schulz, S. Trimper, and M. Schulz, Eur. Phys. J B15, 499 (2000); B. M. Schulz, M. Schulz, and S. Trimper, Phys. Rev. E 66, 031106 (2002).

[12] M. Schulz and S. Trimper, Phys. Rev. B 64, 233101 (2001).

[13] S. Trimper, K. Zabrocki, M. Schulz, Phys. Rev. E 65, 056106 (2002).

[14] S. Trimper, K. Zabrocki, M. Schulz, Phys. Rev. E 66, 026114 (2002).

[15] S. Trimper and K. Zabrocki, Phys. Lett. A 321, 205 (2004).

[16] R. Grima, Phys.Rev.Lett. 95, 128103 (2005).

[17] S. Trimper and . Zabrocki, Phys. Lett. A 331, 423 (2004).

[18] E. Leutheusser, Phys.Rev.A 29, 2765 (1984).

[19] W. Götze in Liquids, Freezing and the Glass Transition edited by Hansen et al. (North Holland, Amsterdam, 1991); for a recent survey, see W.Götze, J.Phys.: Condens. Matter, 11, A1 (1999).

[20] H. Mori, Prog.Theor.Phys. 34, 399 (1965).

[21] M. Schulz, S. Stepanow, Phys. Rev. B 59, 13528 (1999).

[22] T. Ohira, T. Yamane, Phys. Rev. E 61, 1247 (2000).

[23] R. Gerami, Phys. Rev. E 65, 036102 (2002).

[24] S. Fedotov and Y. Okuda, Phys. Rev. E 66, 021113 (2002).

[25] J. D. Murray Mathematical Biology, Part I (Springer, Berlin, 2000).

[26] S. Fritzsche, J. Kärger, Europhys. Lett. 63, 465 (2003). 
[27] D. ben-Avraham and S. Havlin Diffusion and Reactions in Fractals and Disordered Systems, (Cambridge University Press, Cambridge, UK, 2000).

[28] I. Santamaria-Holek and J.M. Rubi, Physica 326, 384 (2003).

[29] V. M. Kenkre, M. N. Kuperman, Phys. Rev. E 67, 051921 (2003).

[30] G. Abramson, A. R. Bishop, and V. M. Kenkre, Phys. Rev. E 64, 066615 (2001).

[31] S. Harris, J. Phys. A: Math. Gen. 36, 8291 (2003).

[32] S. Trimper and K. Zabrocki, Proccedings Spie Fluctuations and Noise (2004).

[33] M. Schulz, B. Schulz, and S. Trimper, Phys. Rev. E 64, 026104 (2001)

[34] P. G. de Gennes, J. Chem. Phys. 76, 3316 (1982).

[35] X. Xing and L. Radzihovsky, Europhys. Lett. 61, 769 (2003).

[36] M. Schulz, S. Trimper and K. Zabrocki, in preparation.

[37] M. Abramowitz and I. A. Stegun, Handbook of Mathematical Functions, (Verlag Harri Deutsch, Frankfurt/Main, 1984).

[38] I.S. Gradshteyn and I.M. Ryzhik, Table of Integrals, Series, and Products, Sixth Ed., (Academic Press, San Diego, 2000). 